\title{
De la enseñanza presencial a la docencia digital. Autobiografía de una historia de vida docente
}

\section{From face to face instruction to digital education. Autobiography of a teaching life}

\author{
Manuel Area Moreira \\ Universidad de La Laguna, La Laguna (España) \\ manarea@ull.edu.es
}

\begin{abstract}
Resumen:
Este artículo describe y analiza el proceso y evolución docente experimentado por el autor a lo largo de dos décadas con relación a innovar su práctica de enseñanza universitaria utilizando Internet. Se apoya en los planteamientos metodológicos narrativos de las denominadas historias de vida y, en particular, en la técnica de la investigación autobiográfica. En esta trayectoria profesional se identifican cinco grandes etapas que han evolucionado desde los primeros espacios web para la enseñanza presencial hasta los actuales desarrollos de la educación a distancia digital. Esta autobiografía no está elaborada de forma intuitiva, sino basada en distintas evidencias empíricas que el autor ha ido recogiendo conscientemente durante los veinte años de duración de la misma. Finaliza el artículo presentando reflexiones teóricas sobre la pedagogía de la educación digital, la influencia del contexto institucional y los cambios culturales de los estudiantes Millennials.
\end{abstract}

\section{Palabras clave}

eLearning, aula virtual, docencia universitaria, educación digital, autobiografía

\begin{abstract}
:
This article describes and analyzes the process and teaching evolution experienced by the author over two decades in relation to innovating his practice of university teaching using the Internet. It is based on the narrative methodological approaches of the so-called life stories and, in particular, on the technique of autobiographical research. In this career, five major stages have been identified that have evolved from the first web spaces for face-to-face teaching to the current developments in digital distance education. This autobiography is not elaborated in an intuitive way, but based on different empirical evidences that the author has been consciously collecting during the twenty years of its duration. The article concludes by presenting theoretical reflections on the pedagogy of digital education, the influence of the institutional context and the cultural changes of Millennials students.
\end{abstract}

\section{Keywords}

eLearning, virtual classroom, higher education, digital education, autobiography 


\section{Introducción}

Desde hace más de una década está proliferado el interés en indagar y explorar nuevas metodologías de investigación social donde se pone el acento en las biografías personales y profesionales de los individuos y no sólo en las representaciones cuantificadas de los comportamientos de grupos humanos más o menos amplios. Una de estas tendencias responde a lo que se conoce como investigación biográfico-narrativa que como señalaron Bolívar, Domínguez y Fernández (2001) "se ha constituido hoy en una perspectiva propia como forma legítima de construir conocimiento en la investigación educativa" (pg. 10).

En lengua española existe un número relevante de publicaciones sobre la experiencia educativa como objeto de estudio (Contreras y Pérez de Lara, 2010), sobre las historias de vida (Ferrarotti, 2007; Medrano, 2007; Hernández, Sancho, y Rivas, 2011; Moriña, 2017), sobre la investigación autobiográfica (Hernández y Rifá, 2011) así como sobre sobre las biografías profesionales de los docentes (Goodson, 2004; Rivas y Herrera, 2009; Rivas, Leite y Prados, 2014). En esta línea también es interesante citar el estudio sobre los incidentes críticos en las trayectorias o biografías de profesorado universitario (Monereo, Montes y Andreucci, 2015).

De forma todavía incipiente están también publicándose algunas investigaciones de naturaleza biográfico-narrativa centrados en el análisis de las historias personales con relación a la tecnología como son el estudio de trayectorias de apropiación tecnológica en jóvenes a través de las denominadas tecnobiografías (Benítez y Lemus, 2016), y los de Sancho (2013) y de Sonlleva, Torrego y Martínez (2017) donde abordan las tecnoautobiografías de estudiantes universitarios en su formación como docentes.

Teniendo en cuenta este marco metodológico de investigación narrativa, muy brevemente esbozado, el objetivo de este artículo es ofrecer una reconstrucción crítica del proceso biográfico vivido por el autor a lo largo de dos décadas con relación a innovar la docencia universitaria utilizando las TIC ${ }^{1}$. Proceso que comencé en 1997 elaborando una página web para una asignatura de docencia presencial y que ha ido evolucionando hasta la actualidad donde desarrollo mi docencia universitaria a través de aulas virtuales combinadas con los recursos de la web 2.0 en un contexto de educación a distancia en línea o en la Red.

La reconstrucción biográfica de este caso docente, a modo de "selfie" profesional $^{2}$, puede servir como ejemplificación del proceso experimentado por un docente universitario en la transición desde modelos de enseñanza presencial hacia la docencia semipresencial, mixta o blearning (Bartolomé, 2008; Barrera, Fernández y Jiménez, 2009) y que, posteriormente, evoluciona hacia prácticas de educación a distancia digital o elearning (Rivera-Vargas; Alonso-Cano y Sancho-Gil, 2017). Esta autobiografía es un relato construido desde el análisis y autoreflexión sobre la

\footnotetext{
${ }^{1}$ Existe poca producción, al menos en lengua española, de informes o artículos autobiográficos de docentes universitarios con relación al uso pedagógico de la tecnología digital. En todo caso, es interesante consultar algunos textos publicados sobre la reconstrucción histórica de las dos últimas décadas sobre la evolución del impacto de la tecnología informática en la educación que aunque no responden a una memoria autobiografíca, en sus páginas están latiendo las experiencias personales de sus autores (Area, 2008; Zapata, 2008).

${ }^{2}$ Utilizo el concepto de "selfie profesional" como una metáfora tomada del fenómeno "selfie" referido a las autofotos o retratos que son compartidos en las redes sociales por el propio autor o autora de los mismos.
}

De la enseñanza presencial a la docencia digital. Autobiografía de una historia de vida docente. Manuel Area Moreira

Página 2 de 21 
experiencia vivida en el uso pedagógico de las TIC en la que se entremezclan conceptos como el aprendizaje mixto o híbrido (Osorio, 2011), la cumplimentación de eactividades (Cabero y Román, 2006), así como en la implementación de un modelo de flipped classroom o aula al revés (Bergmann y Sams, 2014) adaptado a la enseñanza superior (Varios, 2014).

El artículo está organizado en dos grandes bloques o partes diferenciadas. En la primera ofreceré la reconstrucción de mi historia biográfica identificando distintas fases o etapas recorridas de trayectoria profesional. En la segunda parte se presentarán algunas ideas y reflexiones derivadas del diálogo entre la teoría y la experiencia docente narrada.

\section{De la enseñanza presencial a la educación a distancia digital: Reconstrucción autobiográfica de un caso ${ }^{3}$}

A continuación voy a intentar describir, sintetizar y analizar mi historia personal de veinte años como docente en el proceso de apropiación profesional y uso de Internet en la enseñanza universitaria. Está narrada como una historia de vida basada en los distintos "selfies profesionales" que fui recogiendo a lo largo mi experiencia de docente con las tecnologías digitales.

En esta trayectoria identifico cinco etapas claramente reconocibles: una inicial de diseño de recursos on line para la asignatura en formato de página web creada con el lenguaje HTML (periodo 1997-2003). Una segunda, caracterizada por emplear una plataforma LMS donde se crearon aulas virtuales cerradas (periodo 2004-08) con la intencionalidad de experimentar la enseñanza mezclada o de blended learning. Una tercera que responde a una metodología de flipped classroom o aula al revés caracterizada por la creación de vídeos o píldoras audiovisuales y la utilización de los recursos y herramientas de la Web 2.0 (periodo 2009-13). Una cuarta de desarrollo de educación digital totalmente a distancia (periodo 2014-17) y se finaliza con una quinta fase todavía incipiente y que abre los nuevos desarrollos futuros de mi práctica profesional. Estas etapas se sintetizan en el Tabla 1.

\begin{tabular}{|l|l|l|l|}
\hline \multicolumn{9}{|c|}{ Etapas en el proceso de innovación docente con TIC } \\
\hline $1^{\text {a }}$ Etapa & $1997-2003$ & $\begin{array}{l}\text { La página web en } \\
\text { HTML de la asignatura } \\
\text { como apoyo a la } \\
\text { docencia presencial }\end{array}$ & $\begin{array}{l}\text { Enseñanza presencial desarrollada } \\
\text { en aula de informática a través de la } \\
\text { web docente de la asignatura }\end{array}$ \\
\hline $2^{\text {a }}$ Etapa & $2004-08$ & $\begin{array}{l}\text { Blearning a través de } \\
\text { Aula Virtual LMS }\end{array}$ & $\begin{array}{l}\text { Modelo b-learning combinando aula } \\
\text { virtual con clases presenciales }\end{array}$ \\
\hline $3^{\text {a }}$ Etapa & $2009-13$ & $\begin{array}{l}\text { Blearning ampliado: } \\
\text { Aulas Virtual + Web } \\
2.0\end{array}$ & $\begin{array}{l}\text { Modelo b-learning combinando aula } \\
\text { virtual cerrada con recursos abiertos } \\
\text { de la Web 2.0 }\end{array}$ \\
\hline $4^{\mathbf{o}}$ Etapa & $2014-17$ & Educación digital a & Educación a distancia en línea \\
\hline
\end{tabular}

\footnotetext{
${ }^{3}$ Esta primera parte del artículo dedicada a la reconstrucción y análisis autobiográfico está basado en un trabajo previo del autor realizado hace casi una década (Area, 2010) por lo que puede entenderse como una actualización y continuación del mismo.
}

De la enseñanza presencial a la docencia digital. Autobiografía de una historia de vida docente. Manuel Area Moreira 


\begin{tabular}{|l|l|l|l|}
\hline & distancia & $\begin{array}{l}\text { basado en proyectos e interacción en } \\
\text { tiempo real y diferido }\end{array}$ \\
\hline $5^{\circ}$ Etapa & $2018-i ?$ & $\begin{array}{l}\text { Educación digital a a } \\
\text { distancia personalizada }\end{array}$ & $\begin{array}{l}\text { Analíticas de aprendizaje y } \\
\text { personalización de itinerarios de } \\
\text { aprendizaje }\end{array}$ \\
\hline
\end{tabular}

El investigador, narrador o autor de este artículo es el personaje de una historia profesional donde Internet (y específicamente la docencia virtual) juega una doble funcionalidad: ser un espacio digital para la práctica docente y a la vez también ser objeto de estudio.

Esta autobiografía no está elaborada de forma intuitiva, sino basada en distintas evidencias empíricas que el autor ha ido recogiendo conscientemente durante estas dos décadas. Para ello se han utilizado los siguientes instrumentos de recogida de datos:

- Los diarios personales del docente.

- Los duplicados y capturas de pantalla de las aulas virtuales creadas.

- Los resultados de las distintas encuestas de opinión que cada curso académico fueron respondidas por los estudiantes

- Los informes elaborados dirigidos a los servicios de innovación y apoyo virtual de la Universidad de La Laguna ${ }^{4}$.

La etapa inicial del viaje: la página web en HTML de la asignatura como recurso de apoyo a la docencia presencial (1997-2003)

A mediados de los años noventa mi universidad (Universidad de La Laguna. Islas Canarias. España) emprendió la política de dotar a los Departamentos, Centros y Facultades de los recursos e infraestructuras de telecomunicaciones informáticas destinados a la investigación y docencia. En pocos años pasamos de una situación absolutamente precaria de ordenadores a otra en la que prácticamente todos los docentes dispusimos de una máquina digital para el trabajo académico en nuestros despachos. Por otro lado, mi curiosidad e interés tanto personal como profesional ligado a la evolución de las tecnologías digitales propició que empezara a experimentar y aplicar los recursos de Internet en la docencia de la asignatura que impartía denominada Tecnología Educativa. Fue en octubre de 1997 cuando elaboré el primer website educativo elaborado con un software que permitía crear páginas web en lenguaje HTMLdestinado a apoyar o complementar mi actividad docente de esta materia

Esta primera versión de una web sobre los contenidos de la asignatura (ver imagen 1) adoleció de algunas limitaciones y errores derivadas de la ingenuidad y falta de experiencia en la creación de este tipo de materiales didácticos distribuidos a través de la WWW. Sin embargo, he de reconocer que el proceso de creación del mismo sirvió para que adquiriera los conocimientos y destrezas necesarias en el diseño de hipertextos

\footnotetext{
${ }^{4}$ Estos informes se corresponden a las memorias finales que fueron elaborados como parte de los proyectos de innovación docente en los que participé tanto convocados por la Unidad de Docencia Virtual como por Vicerrectorado de Docencia de la Universidad de La Laguna desde 2005 hasta 2017.
}

De la enseñanza presencial a la docencia digital. Autobiografía de una historia de vida docente. Manuel Area Moreira

Página 4 de 21 


\section{Imagen 1}

$1^{a}$ versión de la web de Tecnología Educativa. Octubre 1997
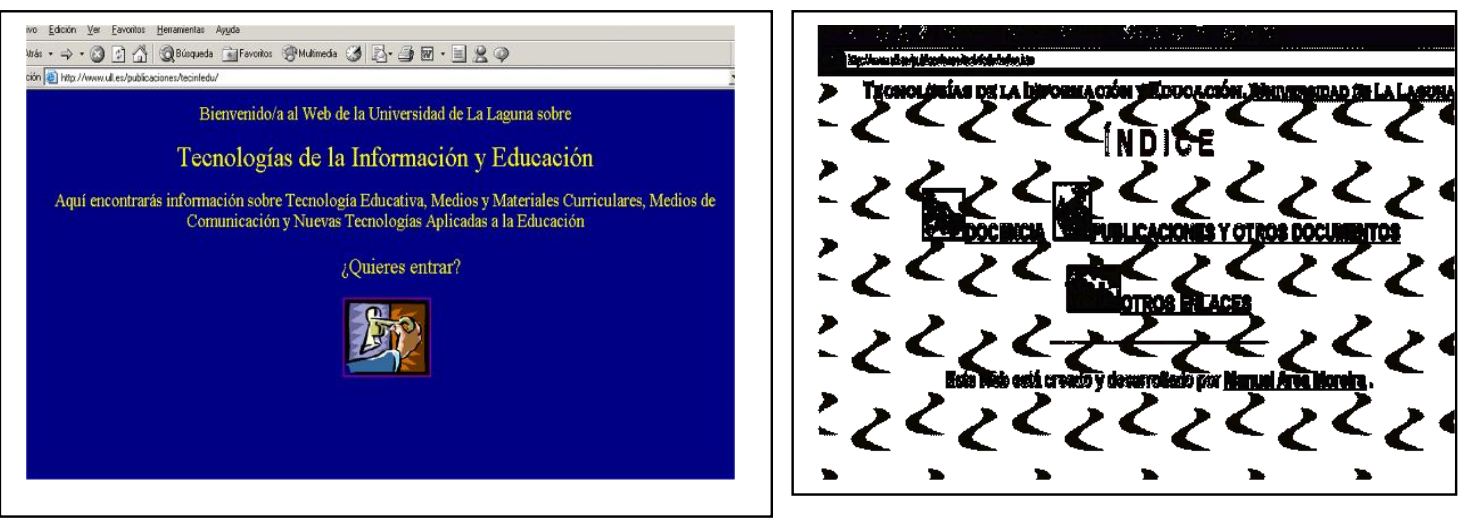

así como para teorizar en torno a los componentes que debiera incorporar un sitio web con fines educativos. En esta primera versión no separé lo que era un material destinado a mis alumnos sobre la asignatura con lo que era difusión de mis trabajos de investigación y publicaciones.

Año y medio después (en enero de 1999) diseñé, desarrollé y publiqué lo que constituyó la segunda versión de la web de la asignatura, aunque en esta ocasión sí se podría decir que se había creado un website propiamente educativo y útil para la docencia y el aprendizaje (Ver imagen 2).

Imagen 2:

$2^{a}$ versión del web de Tecnología Educativa. Enero 1999

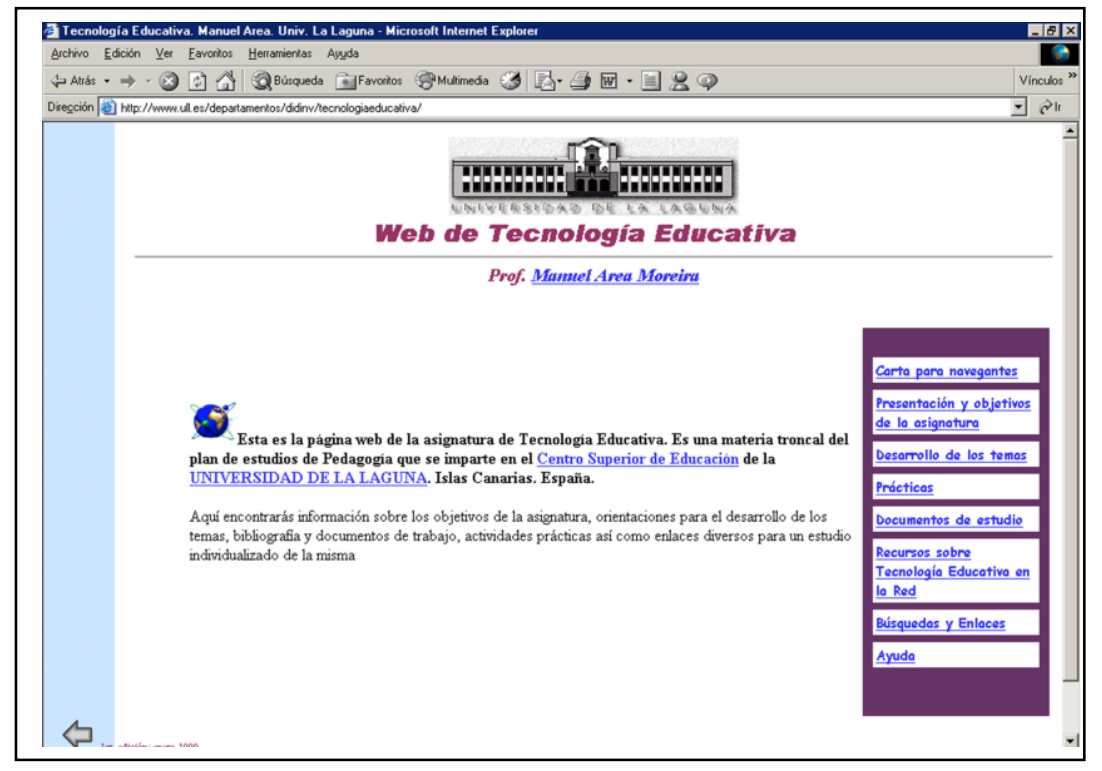

Esa web puede considerarse como una de las experiencias pioneras en el contexto de la docencia universitaria en España en cuanto que la misma se diseñó no como una web de naturaleza informativa, sino como una web concebida como material didáctico, es decir, pensada y desarrollada para que el alumnado pudiera cursar la asignatura a través de la misma. Este website fue un material diseñado

De la enseñanza presencial a la docencia digital. Autobiografía de una historia de vida docente. Manuel Area Moreira

Página 5 de 21 
hipertextualmente proporcionando al alumnado información relativa al programa de la asignatura (los objetivos, el temario, la bibliografía, las prácticas); los documentos completos que le permitieran estudiar los contenidos en su hogar, una selección de enlaces con otros webs sobre la misma materia tanto en España como en otros países.

La estructura de aquella web estaba configurada por las siguientes opciones:

- Presentación y Objetivos de la Asignatura

- Desarrollo de los temas

- Prácticas

- Documentos de Estudio,

- Recursos de Tecnología Educativa en la Red

- Búsqueda y Enlaces

Tenemos que añadir que en esta primera versión (curso 1998-1999) no se incluyeron recursos telemáticos de comunicación. Al año siguiente, es decir, en el curso 1999-2000 se crearon dos foros de debate. En el curso 2001-02, y como consecuencia de un proyecto de $\mathrm{I}+\mathrm{D}$ denominado Internet en la docencia universitaria. Diseño y experimentación de material didáctico multimedia distribuido a través de la WWW financiado por la Dirección General de Universidades e Investigación del Gobierno de Canarias fue posible configurar un grupo de trabajo centrado en la producción de material docente multimedia distribuido a través de Internet. Uno de los principales objetivos de dicho proyecto consistió en rediseñar y desarrollar un nuevo website para la asignatura de Tecnología Educativa y experimentarlo con grupos reales de alumnado.

Esta tercera versión del website de la asignatura partió de una concepción semipresencial de la docencia: es decir, para el desarrollo de actividades docentes en el sentido tradicional universitario (encuentro físico del profesor y un grupo de alumnos en un mismo espacio físico), y para el desarrollo de actividades autónomas por parte del

$3^{\text {a }}$ versión de la web de Tecnología Educativa. Marzo 2002

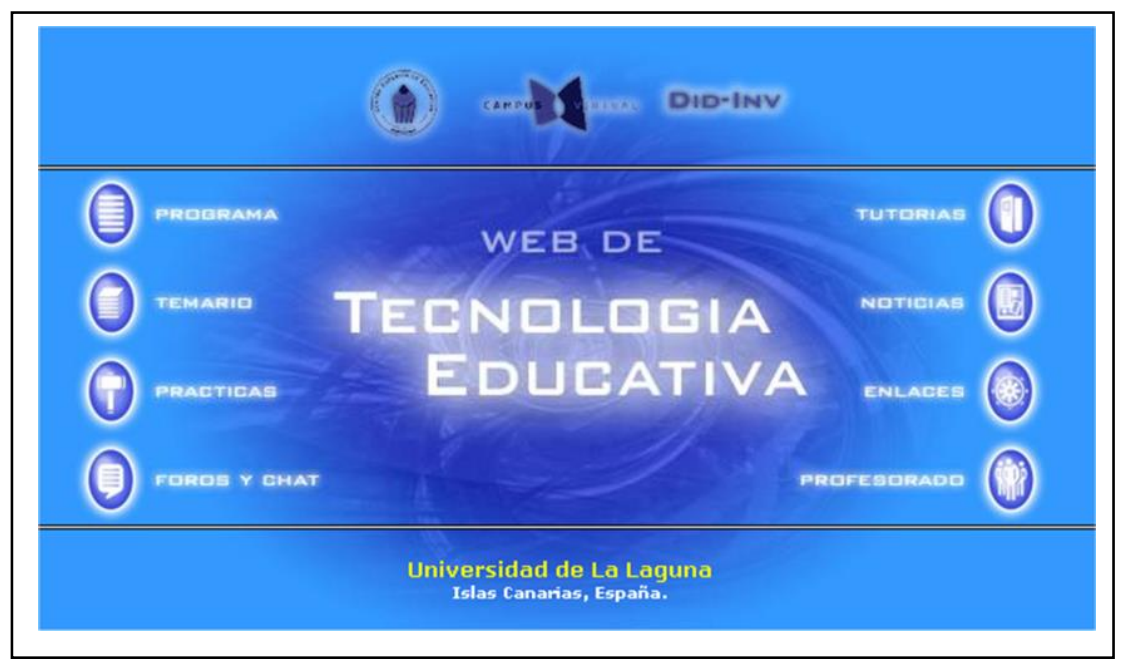

alumnado (en su casa, en un cibercafé o en el aula de informática de la Facultad) sin la presencia física del docente. Esto representó un importante replanteamiento o innovación del papel de la tecnología en la metodología didáctica ya que significó que

De la enseñanza presencial a la docencia digital. Autobiografía de una historia de vida docente. Manuel Area Moreira

Página 6 de 21 
el proceso de enseñanza-aprendizaje de esta asignatura se apoyaba en situaciones presenciales y a distancia de uso de este material. Por ello, en el rediseño del sitioweb se incorporaron las siguientes dimensiones o elementos: los objetivos de la asignatura, un manual electrónico con el desarrollo de los contenidos de todos los temas presentado en formato PDF, las diapositivas/presentaciones utilizadas en las exposiciones teóricas elaboradas en Power Point, los recursos necesarios para la realización de las prácticas, documentos completos de estudio y ampliación de conocimientos de la asignatura, un conjunto de links o enlaces seleccionados sobre la educación y las nuevas tecnologías, foros de debate virtuales específicamente creados para cada grupo de alumnos de la asignatura, y el correo electrónico de los docentes de la misma.

Hoy en día, incorporar estos elementos parece una acción normal y necesaria, pero en aquellos años no era habitual encontrarlo. Quizás por ello, esa versión del sitioweb de la asignatura recibió el primer premio en 2003 en el I Certamen Intercampus organizado por la Fundación Telefónica.

\section{La segunda etapa: El b-learning a través de un aula virtual LMS (2004-2008)}

En abril de 2004 el Vicerrectorado de Planes de Estudio y Títulos Propios la Universidad de La Laguna convocó un concurso público para Proyectos Piloto de experimentación del Crédito Europeo. En dicha convocatoria se establecía que el principal objetivo era el profesorado experimentase la puesta en práctica del nuevo modelo educativo propuesto por la Declaración de Bolonia y la obtención de resultados académicos que conformen una opinión de la comunidad universitaria tanto en la forma de desarrollar las enseñanzas como en la reestructuración de las titulaciones.

Teniendo en cuenta el contexto de experimentación de los créditos europeos junto con la necesidad de experimentar un proceso de enseñanza apoyado en el uso de las TIC (Tecnologías de la Información y Comunicación) en general, y específicamente un software para la teleformación o eLearning, como es la plataforma MOODLE, en junio de 2004 inicié el proceso de diseño del "aula virtual" para la asignatura de Tecnologías de la Información y Comunicación en Educación, del título de Pedagogía de la Facultad de Educación de la Universidad de La Laguna (Ver imagen 4).

La principal innovación metodológica desarrollada en esta asignatura respecto a cursos anteriores consistió en desarrollar una metodología plenamente semi-presencial, mixta o híbrida de enseñanza (blended learning) que combinara distintas actividades docentes desarrolladas a través de un aula virtual específicamente creada para esta asignatura, y sesiones presenciales en la clase habitual. Esta aula virtual docente se gestionó bajo la plataforma MOODLE 1.4.1 para el seguimiento y apoyo a las actividades tanto teóricas como prácticas. Es necesario indicar, que en aquel tiempo, mi universidad no disponía de un servicio o unidad de Campus Virtual. Por ello, la instalación y gestión de esta plataforma LMS estaba ubicada en el servidor de mi propio grupo de investigación EDULLAB (Laboratorio de Educación y Nuevas Tecnologías) http://edullab.webs.ull.es/

De la enseñanza presencial a la docencia digital. Autobiografía de una historia de vida docente. 
Imagen $n^{\circ} 4$

Aula Virtual de la asignatura en Moodle (2004)

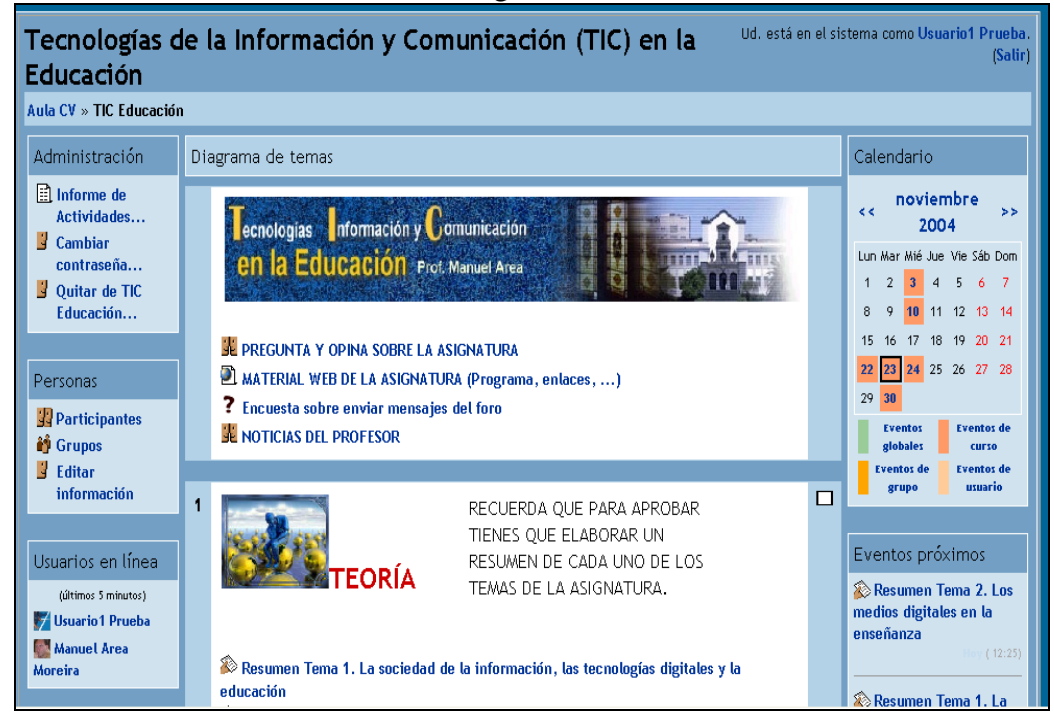

La metodología de enseñanza desarrollada fue un modelo mixto que combinaba sesiones presenciales (bien en el aula de informática de la facultad, bien en la clase habitual) con otras a través del aula virtual. Aproximadamente la distribución del tiempo fue del $50 \%$ para sesiones presenciales, y $50 \%$ para la docencia virtual.

El proceso de trabajo que debía desarrollar el alumnado se basó en el desarrollo de:

- prácticas que los estudiantes tenían que cumplimentar a través del aula

- elaboración y envío al aula virtual de un resumen de cada uno de los siete temas que configuran el manual de estudio de la asignatura

- participación en los foros y otros recursos creados dentro del aula virtual

- asistencia a las sesiones presenciales que se desarrollaron a modo de seminarios de discusión sobre los temas teóricos.

Las prácticas fueron el eje central del proceso autónomo de aprendizaje del alumnado y la comunicación a través del foro de debate de la clase fue el principal instrumento de comunicación entre estudiantes y docentes en dicha aula virtual. El apoyo docente o tutorización al alumnado se desarrolló combinando dos situaciones/modalidades de comunicación entre docente y discentes: a través del aula virtual; y en el despacho del profesor.

La experiencia iniciada con las aulas virtuales Moodle fue, en líneas generales, altamente valorada por los estudiantes a través de test o cuestionarios de opinión que pasaba al final del curso. Para mi práctica docente representó un salto cualitativo importante respecto a las antiguas webs de la asignatura: por una parte, obtenía información automatizada sobre las acciones desempeñadas por mis estudiantes (participación en foros, entradas y actividades dentro del aula virtual, mensajería interna, subida y entrega de trabajos, etc.); y por otra, la integración de distintas herramientas informáticas -foros, archivos de texto, enlaces, presentaciones, ...- en un único entorno virtual, lo que facilitaba enormemente la creación de los cursos o aulas virtuales específicas para cada asignatura.

De la enseñanza presencial a la docencia digital. Autobiografía de una historia de vida docente. Manuel Area Moreira 
La tercera etapa: B-learning desarrollado más allá del aula virtual cerrada: $L M S+W e b 2.0(2009-13)$

La tercera etapa se desarrolla entre los cursos 2009-13. Es una fase caracterizada por el uso combinado de aulas virtuales ubicadas en el campus virtual de mi universidad con otros recursos de la web 2.0 así como en la utilización de píldoras audiovisuales o videolecciones para facilitar un modelo de flipped classroom. La aparición y crecimiento de los recursos de la denominada web 2.0 -los blogs, las wikis y las redes sociales- junto con la creación del servicio de grabación audiovisual ULLMedia en mi universidad provocó un nuevo punto de inflexión para mi práctica docente. En este sentido, mi preocupación consistió en cómo combinar lo que es el aula virtual como un espacio cerrado y privado entre mis estudiantes y su profesor con los recursos abiertos y públicos de la web 2.0 cara a cumplimentar nuevas tareas de aprendizaje.

En la planificación de estas nuevas herramientas seleccioné el uso de los blogs, las wikis y los repositorios/bibliotecas de archivos audiovisuales y multimedia (especialmente procedentes de YouTube y de SlideShare). De esta experiencia, confirmé que la Web 2.0 tienen un potencial enorme en la docencia universitaria ya que:

a) Las herramientas de la web 2.0 ofrecen recursos potentes para que los estudiantes puedan cumplimentar tareas que no podrían ser realizadas sin las mismas de forma rápida, barata y sin requerir conocimientos informáticos. Entre estas tareas destacaría aquellas vinculadas con la "publicación" (sea de documentos de texto, de presentación multimedia, de video, de sonido, de blog, ...).

b) La web 2.0, en el contexto de la docencia universitaria, sirve para favorecer el desarrollo de las competencias digitales, informacionales y de trabajo colaborativo entre los estudiantes.

c) La web 2.0 permite hacer visible, público y abierto los distintos productos de los estudiantes lo que favorece que éstos vayan conociendo y adquiriendo los valores de la filosofía del conocimiento compartido y de la inteligencia colectiva.

d) La web 2.0 ofrece una enorme y variada riqueza de objetos o materiales de aprendizaje generados por otros docentes, instituciones o usuarios que pueden ser reutilizados pedagógicamente para los objetivos formativos de nuestras materias.

De la enseñanza presencial a la docencia digital. Autobiografía de una historia de vida docente. 
Imagen 5: Aula virtual asignatura título Maestro Primaria (2012)

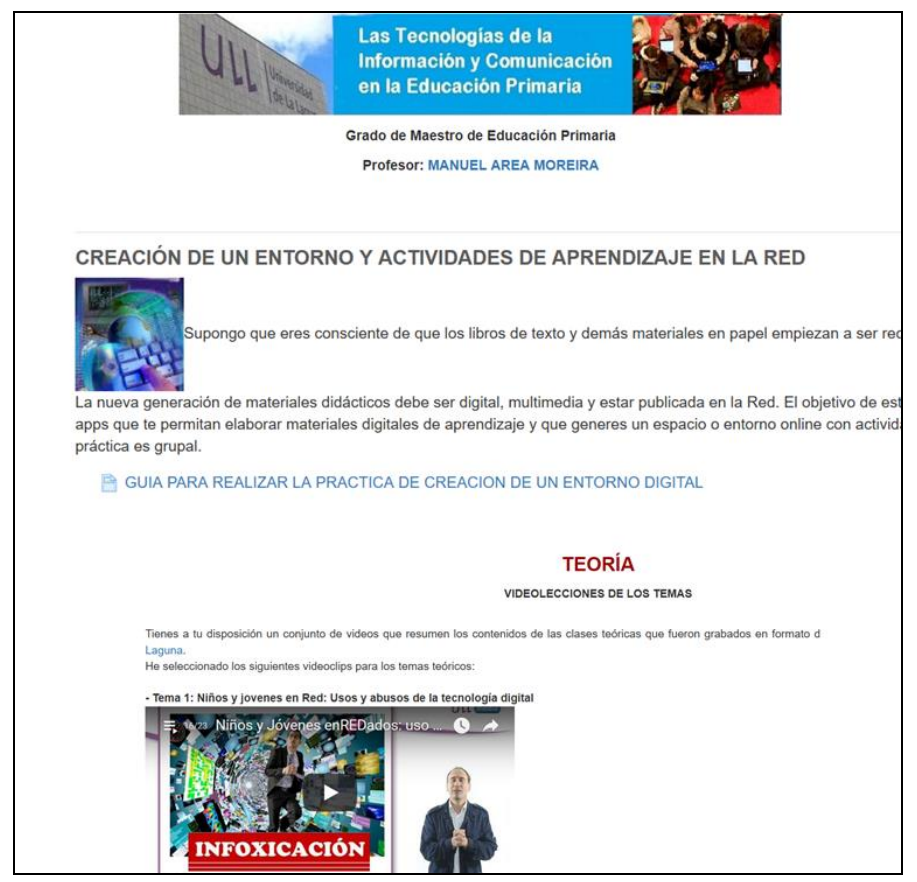

En este sentido, la combinación Moodle+Web 2.0+Videolecciones (ver imagen 5) permitió que el aula virtual dejara de ser un "jardín vallado" o espacio cerrado, para empezar a utilizar toda la Red como plataforma (Area y Adell, 2009). Asimismo, las innovaciones más destacadas con relación a los procesos de aprendizaje consistieron en:

- Incrementar el conjunto de tareas y actividades que permitieran un mayor grado de autonomía en el alumnado de modo que el desarrollo de sus prácticas no sean siempre tuteladas bajo la dirección de un docente. En este sentido, el concepto de PLE (Personal Learning Enviroment) (Attwell, 2007; Castañeda y Adell, 2013; Area y Sanabria, 2014; Coll, Engel, Saz, y Bustos, 2014) y de e-portafolio (Barberá y otros, 2006; Fraga y Gewerc, 2009) como herramienta de trabajo del estudiante y para el seguimiento y evaluación del aprendizaje de los mismos fue un referente en mi práctica docente. Sin embargo, la experiencia de utilización de un software específico para los portafolio electrónicos -como fue el caso de Mahara- no funcionó existosamente ya que el alumnado percibió una doble tarea en su quehacer online: la desarrollada a través del aula Moodle y del espacio de dicho e-portafolio.

- Aumentar las demandas de trabajo colaborativo o en equipo entre el alumnado utilizando los recursos informáticos. Las wikis y el software de redes sociales se configuraron como herramientas vinculadas a las tareas desarrolladas con el aula virtual Moodle.

- Seguir potenciando el proceso de aprendizaje constructivista y basado en la realización de e-actividades. En este sentido, el diseño pedagógico de las aulas virtuales en esta fase fue planteado desde una organización de aprendizaje por tareas, no de organización temática por contenidos.

- Reconvertir el tiempo docente de la clase presencial en un espacio para el seguimiento y tutorización de la actividad práctica del alumnado y no para la oferta de contenido teórico ya que éste estaba presentado en videolecciones incluidas en el aula virtual.

De la enseñanza presencial a la docencia digital. Autobiografía de una historia de vida docente. Manuel Area Moreira

Página 10 de 21 


\section{La cuarta etapa: La educación a distancia digital (2014-17)}

Durante el curso 2012-13 tuve la oportunidad de elaborar el diseño de la primera titulación oficial de mi universidad ofertada totalmente a distancia empleando los recursos de Internet: Máster en Educación y Tecnologías de la Información y Comunicación (MEDUTIC) $)^{5}$. A partir del curso 2014-15 empezó a impartirse por lo que concentré mi atención en reformular mi docencia para adecuarla a las exigencias de lo que suponía la docencia a distancia digital.

Imagen 6 Aula virtual Master (2016-17)

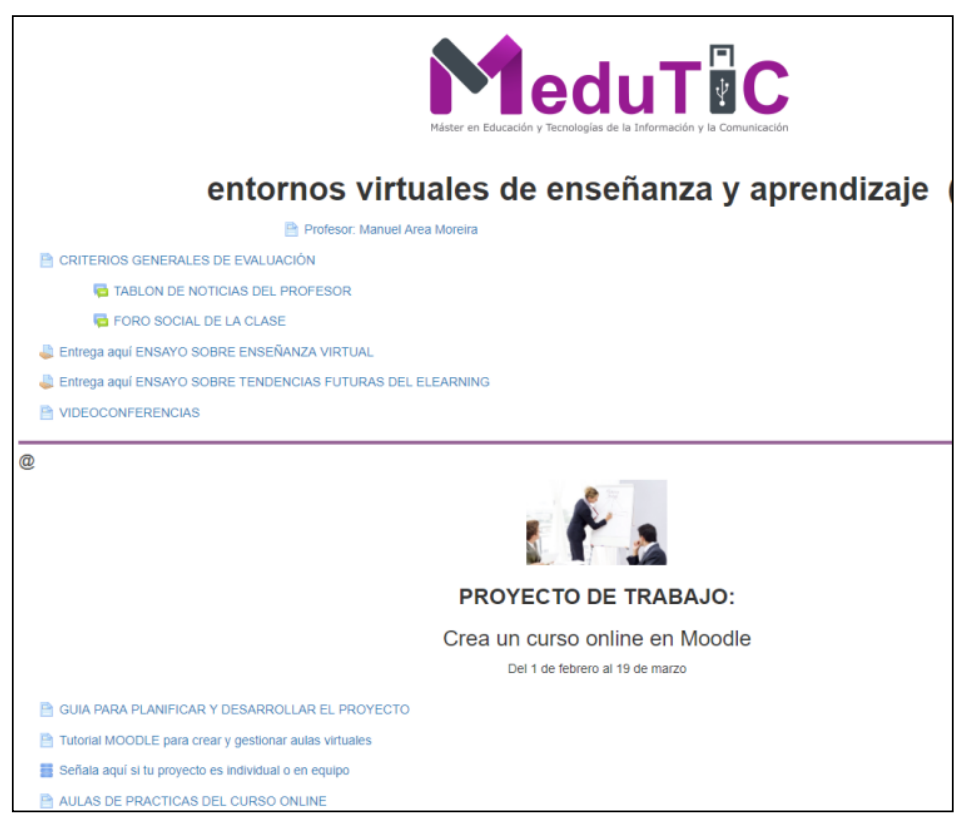

El planteamiento fue continuar con el modelo de aula virtual ya experimentado en los cursos anteriores, aunque incorporando las siguientes variantes (ver imagen 6):

- $\quad$ El eje organizativo de la asignatura consistió en focalizar el proceso de trabajo del alumnado durante la asignatura en torno a un proyecto de trabajo amplio lo más próximo a lo que sería una futura tarea profesional. En otras palabras, la principal demanda del aprendizaje consistía en la realización de una macrotarea o proyecto de aprendizaje auténtico. Está podía ser abordada en solitario o en equipo.

- Paralelamente se ofreció una síntesis de los contenidos básicos mediante videoclips didácticos o videolecciones expresamente creados para la materia donde se solicitó tareas individuales de reconstrucción y ampliación de conocimiento.

- Creación de espacios de comunicación e interacción social continuada entre los estudiantes, y el docente. Estos espacios adoptaron el formato de videoconferencias en tiempo real, grabadas y puestas a disposición en el aula virtual para su visualización permanente.

- Evaluación continuada con feedback en la entrega de las distintas tareas que tenían que elaborar los estudiantes.

La experiencia fue altamente positiva aunque, como suele suceder en esta modalidad educativa, el grado de implicación y éxito del alumnado fue variado ya casi

\footnotetext{
${ }^{5}$ Aprobado en BOE no 104 de 29 de abril de 2014

De la enseñanza presencial a la docencia digital. Autobiografía de una historia de vida docente. Manuel Area Moreira

Página 11 de 21
} 
un tercio de los mismos que no pudo cumplimentar en tiempo y forma las tareas demandadas. Curiosamente este porcentaje fue similar en los tres cursos académicos de dicha experiencia docente.

De forma paralela con el surgimiento y expansión de los MOOC (Cursos Masivos Abiertos en Línea) tuve la oportunidad de diseñar y desarrollar los materiales didácticos de varios de estos cursos masivos. En concreto, desarrollé dos cursos MOOC de formación del profesorado para el $\mathrm{INTEF}^{6}$, y uno para la oferta que realiza mi universidad. Ello me dio la oportunidad para conocer y experimentar esta otra modalidad formativa a distancia de educación no formal. Quizás la aportación más interesante tenga que ver con la experimentación en la creación de microcontenidos audiovisuales y en la planificación de microtareas que tienen que cumplimentar los participantes en un curso MOOC.

\section{Empezando una nueva etapa: La educación a distancia personalizada (2018-¿?)}

Probablemente los siguientes pasos a desarrollar en mi experiencia docente estén vinculados a los nuevos desarrollos tecnológicos basados en las analíticas del aprendizaje y a los procesos de personalización y adaptabilidad a las características individuales del alumnado. De hecho, se abre la oportunidad de explorar y experimentar con una nueva generación de $\mathrm{LMS}^{7}$ que, basada en MOODLE, representa un avance en sus limitaciones como espacio virtual para la enseñanza ${ }^{8}$. Mi intencionalidad es desarrollar un nuevo entorno formativo digital caracterizado por:

- Presentar una interface más gráfica y visual, lo que representaría una mejora respecto a mis anteriores aulas virtuales donde predominaban los elementos textuales.

- Disponer de una gestión automatizada más completa de los datos e informaciones de la actividad de los estudiantes, a modo de analíticas de aprendizaje que me permitan tener un conocimiento más profundo de la actividad online de cada estudiante y realizar seguimiento del mismo.

- Desarrollar un entorno que ofrezca mayor flexibilidad y adaptabilidad del diseño didáctico del curso y por tanto personalización de los itinerarios y tareas que pueden cumplimentar los estudiantes. De este modo, dejaría de ofrecer un entorno estandarizado de aprendizaje igual para todos, para crear itinerarios de contenidos y de actividades diferenciadas entre grupos de estudiantes.

En síntesis, los nuevos pasos a dar son: consolidar el aprendizaje auténtico a través de proyectos o macrotareas que tienen que desarrollar el alumnado empleando una variedad de aplicaciones y recursos disponibles en el ciberespacio, incrementar la comunicación e interacción en tiempo real y diferido, generar nuevos materiales en formato de microcontenidos multimedia y audiovisuales, emplear las analíticas del aprendizaje cara a realizar seguimiento de la actividad y progreso de los estudiantes, y

\footnotetext{
${ }^{6}$ MOOCs para INTEF “Enseñar y evaluar la competencia digital” (4 ediciones), "Enseñar en la escuela digital: la competencia digital docente" (en producción). MOOC para la ULL "“Nuevas tendencias en educación y TIC"

${ }^{7}$ Vease el concepto de NGDLE (Next Generation Digital Learning Environment) propuesto por Brown, Dehoney y Millichap, 2015.

${ }^{8}$ Me refiero, a modo de ejemplo, a la plataforma MOODLEROOMS https://es.moodlerooms.com/
}

De la enseñanza presencial a la docencia digital. Autobiografía de una historia de vida docente. 
establecer itinerarios de aprendizaje diversificados y, en la medida de lo posible, personalizados.

\section{2. ¿Qué he aprendido? Dialogando entre la experiencia y la reflexión teórica}

Toda autobiografía es la reconstrucción narrada de un viaje personal. Es la memoria escrita de la trayectoria vital de un sujeto donde la experiencia vivida y el análisis reflexivo se entremezclan. Por lo que, a continuación, intentaré dialogar entre la teoría y la experiencia anteriormente expuesta.

Básicamente, lo que pretendo es reflexionar conceptualmente con el intento de responder a la siguientes preguntas ¿qué he aprendido o qué me ha aportado esta biografía para formular nuevo conocimiento pedagógico? ¿qué aproximaciones teóricas con relación a la educación o formación a través de entornos digitales puedo inferir desde mi trayectoria o experiencial profesional como docente? ¿qué características debieran tener los entornos o aulas virtual para desarrollar una pedagogía favorecedora del aprendizaje activo de mis estudiantes?

Estas respuestas las he agrupado en tres reflexiones que giran en torno al paradigma pedagógico de las aulas virtuales, a la influencia del contexto organizativo institucional y a los cambios culturales que ha experimentado el alumnado como primera generación plena de la sociedad digital.

\section{La pedagogía activa como base para enseñar y aprender en la Red}

Lo primero es que estamos asistiendo a un cambio de paradigma dominante en los procesos de enseñanza en general, y específicamente los de educación superior. Este proceso es evidente en los espacios formativos virtuales. Así lo atestiguan, desde hace tiempo, distintos autores ya que los fenómenos que acompañan a la transformación digital de las acciones formativas (y que previsiblemente serán mucho mayores en los próximos años) provocará, según algunos autores, una reforma pedagógica de gran envergadura que ya ha comenzado. Al respecto es interesante consultar los diversos informes que se están publicando a nivel internacional al respecto como son los de Bates (2015); Johnson y otros (2016); Siemens; Gasevic y Dawason (2015); ZawackiRichter y Anderson (2014).

En el contexto español también contamos con una destacable fundamentación de las bases pedagógicas de la enseñanza o educación virtual en los que destacan una serie de textos como Duart y Sangrá (2000); Aguaded y Cabero (2002; 2013); Adell (2004); Cabero (2006); García Aretio (2007); Barberá (2008); Coll y Monereo (2008) por citar algunos de los más referenciados. Estos textos siempre han sido los principales referentes que subyacían y daban corpus teórico a mis prácticas de enseñanza con la tecnología y, en todos ellos, se apuesta por modelos de creación de entornos de aprendizaje digitales que tengan como base teórica la pedagogía activa, la psicología constructivista y el aprendizaje social ${ }^{9}$.

A diferencia de la enseñanza presencial apoyada en la interacción cara a cara con el docente, la educación a en línea o digital se basa en el supuesto de que el alumnado mediante un dispositivo tecnológico conectado a la red - desarrolla procesos de

\footnotetext{
${ }^{9}$ Una teoría educativa sobre la enseñanza y aprendizaje virtual, además de las aportaciones del conocimiento pedagógico y psicológico, también debe incorporar y tener en cuenta los conocimientos desarrollados en el campo de la informática, de la comunicación, de las organizaciones sociales y de la cultura y el conocimiento en la sociedad digital.
}

De la enseñanza presencial a la docencia digital. Autobiografía de una historia de vida docente. Manuel Area Moreira 
autoaprendizaje en el contexto de su hogar o del centro educativo sin las limitaciones de horarios concretos de clase. Para ello es indispensable la existencia de un entorno, aula o escenario virtual donde el estudiante encontrará un conjunto de materiales o recursos didácticos de estudio y trabajo, tareas de aprendizaje, herramientas comunicativas y procedimientos de evaluación. Un entorno de formación se refiere siempre a un lugar reconocible en el ciberespacio que posee una identidad y estructura definida con fines educativos. Un entorno formativo digital siempre es creado con la intencionalidad de estimular, guiar o supervisar un proceso de aprendizaje (Area y Adell, 2009).

Evidentemente pueden existir distintas variantes de aulas o entornos formativos on line oscilando entre un enfoque abierto y flexible o una estructura formativa cerrada y rígida. Desde mi punto de vista, el grado de estructuración y flexibilidad de un entorno formativo virtual vendrá dado por dos factores: el planteamiento o modelo didáctico de dicho entorno (existen un continuum de estrategias didácticas que van desde un planteamiento expositivo de la información hasta otras de descubrimiento libre); y por el tipo de herramientas digitales utilizadas (existe un amplio abanico de posibilidades de plataformas LMS, de recursos online abiertos o de aplicaciones en el ciberespacio que permiten crear aulas o entornos específicamente de naturaleza formativa). Estos entornos en línea pueden estar encerrados en sí mismos, o bien abiertos y distribuidos por todo Internet.

Teniendo en cuenta los referentes teóricos citados y lo aprendido a lo largo de mi práctica docente podemos afirmar que existen dos grandes modelos o enfoques pedagógicos para la creación y uso de aulas o entornos virtuales para la formación:

a) Un enfoque de aulas virtuales basado en el paradigma de la pedagogía expositiva caracterizado por concebir los entornos online como espacios de presentación del conocimiento empaquetado en objetos digitales para el aprendizaje por recepción, así como por el desarrollo de procesos de enseñanza y aprendizaje individuales y estandarizados para todos.

b) Una enfoque de aulas virtuales basado en el paradigma de la pedagogía activa o experiencial donde éstas se conciben como espacios organizados en función de la demanda de cumplimentación de actividades, tareas y proyectos que el alumnado tiene que desarrollar en interacción con otros estudiantes empleando distintos objetos y recursos en línea, así como en la flexibilidad y adaptabilidad a los ritmos y necesidades personales de los estudiantes.

De modo similar, Mauri y Onrubia (2008) coinciden en identificar también dos grandes concepciones del proceso de enseñanza virtual: una centrada en la dimensión tecnológica, y otra en la construcción del conocimiento por los estudiantes.

A lo largo de mi biografía docente ambos enfoques o pedagogías han estado implícitos. He de reconocer que el enfoque de la pedagogía expositiva estuvo en la base de mis primeras experiencias de creación de entornos formativos digitales, pero a medida que fui consolidando mi experiencia docente con la tecnología fui concienciándome y articulando propuestas de enseñanza-aprendizaje en línea con una decidida apuesta hacia concebir los entornos o aulas desde un enfoque de pedagogía activa y de aprendizaje experiencial, aunque sin renunciar a la incorporación de objetos digitales portadores de conocimiento.

De la enseñanza presencial a la docencia digital. Autobiografía de una historia de vida docente. 


\section{La poderosa influencia del contexto institucional}

En segundo lugar, también he aprendido que toda biografía profesional de un docente se produce en un contexto institucional que lo condiciona bien para estimular su práctica innovadora, bien para neutralizarla. Por ello otra conclusión a extraer de la experiencia biográfica vivida tiene que ver con la génesis y expansión de la docencia virtual o en línea en el contexto de la universidad española en estos veinte años. Cuando comencé a experimentar con las TIC en mi docencia -a finales del siglo pasado (en la década de los noventa)- Internet era un fenómeno desconocido entre la mayoría de los docentes universitarios. Hoy en día, por el contrario, la enseñanza o docencia virtual es una realidad extendida y consolidada, en mayor o menor medida, en todas las universidades tanto del ámbito español como internacional.

A lo largo de estas últimas dos décadas las autoridades y gestores universitarios han impulsado y apoyado institucionalmente la incorporación de las TIC a la docencia universitaria. Desde hace varios años, la CRUE -Conferencia de Rectores de las Universidades Españolas- publica anualmente su informe sobre Las Tecnologías de la Información y las Comunicaciones en el sistema universitario español (UNIVERSITIC) en el que describe y analiza la situación de implantación y desarrollo de las tecnologías digitales en el contexto universitario. En el último informe UNIVERSITIC (Gómez Ortega, 2016) se afirma:

"Un año más, el informe corrobora la apuesta de las universidades por las TI como soporte y apoyo a la docencia, mostrando una consolidación de la tendencia de los últimos años... La docencia virtual, la gestión de licencias software para docencia y el soporte a aulas de informática de uso docente están implantados prácticamente en la totalidad de las universidades." (pg. 12).

Este hecho representa un contexto institucional que está impulsando decididamente la experimentación y desarrollo de nuevos formatos de docencia universitaria lo que supone un impulso y extensión de modelos híbridos o semipresenciales de enseñanza de titulaciones ofertadas a distancia así como de los MOOCs como un tipo de oferta formativa de muchas universidades españolas tal como atestigua el informe UNIVERSITIC antes citado.

Sin embargo, queda todavía mucho camino por recorrer en el proceso de integración plena y de transformación digital de la docencia en educación superior tanto en la modalidad de educación presencial como en la educación a distancia. Entre las estrategias y acciones a implementar sería necesario implementar acciones de naturaleza organizativa-pedagógica como las siguientes:

- reformular los modelos organizativos tradicionales de la universidad del siglo XX (horarios, espacios físicos, normas y reglas académicas, ...) con los nuevos modelos de organización universitaria del S. XXI

- superar las resistencias y actitudes poco favorables hacia la educación digital en sus variadas formas tanto en docentes, estudiantes y equipos directivos universitarios,

- reelaborar las normativas o reglamentos universitarios que regulan la dedicación horaria docente adecuándolos a las necesidades y características de los contextos de enseñanza híbrida, mixta y a distancia,

- cambios en la metodología de enseñanza, donde prime más el aprendizaje por tareas y proyectos desarrollados autónomamente por los estudiantes que una metodología expositiva de lecciones magistrales,

- flexibilizar los horarios de clases presenciales e incrementar los tiempos de

De la enseñanza presencial a la docencia digital. Autobiografía de una historia de vida docente. Manuel Area Moreira

Página 15 de 21 
trabajo y aprendizaje a través de los espacios o entornos formativos online (esto evidentemente deberá ir acompañado con la mejora de la conectividad y acceso a la red desde cualquier sitio del campus),

- reconfigurar los roles y funciones del profesor de forma que cobre más relevancia sus competencias en la creación de materiales didácticos digitales, la organización y gestión de entornos o aulas virtuales, la comunicación y tutorización a través de recursos online, la impartición de clases online mediante videoconferencia, entre otras,

- redefinir las funciones y utilidades de los espacios físicos de los centros universitarios de modo que exista mayor disponibilidad de seminarios, bibliotecas, salas de reuniones, aulas, espacios o talleres maker, etc. para que el alumnado pueda reunirse y trabajar autónomamente sin la presencia de su profesor, pero conectados a los entornos educativos online.

\section{El alumnado ha cambiado: la generación de los Millennials}

A lo largo de esta historia de vida docente de veinte años el contexto cultural, tecnológico e imaginario de expectativas e intereses de mis estudiantes se ha transformado radicalmente. Cuando empecé a utilizar Internet en mi docencia a finales del siglo XX la mayoría de mi alumnado desconocía lo que era el ciberespacio y la mayoría no poseía un ordenador o tecnología propia. Su experiencia tecnológica se reducía al consumo de la televisión, el cine y los videojuegos. De hecho, muchos de ellos planteaban resistencias a la incorporación y uso de aulas o entornos virtuales en las prácticas que les demandaba.

Hoy en día el panorama es bien distinto y los efectos de la omnipresencia tecnológica en las vidas cotidianas de los jóvenes han trastocado sus pautas y prácticas de conducta social y cultural. Sobre este particular existe actualmente una muy notoria producción de estudios e informes sobre los cambios que las tecnologías móviles y las redes sociales están provocando en el comportamiento y consumo de la cultura en la denominada generación de los Millennials. En otra ocasión tuve oportunidad de analizar con mayor detalle los principales rasgos o manifestaciones de jóvenes con relación a la utilización del ecosistema digital (Area, Borrás y Sannicolás, 2015).

En dicho trabajo señalábamos que los Millennials pudieran considerarse como la primera generación realmente nacida en el contexto de la sociedad de la información y/o digital. Son una generación expuesta e inmersa en el sobreuso de los medios tecnológicos, de exceso de estimulación sensorial a través de imágenes y sonidos, que reciben diariamente ingentes cantidades de información desde múltiples fuentes y recursos, que están en permanente contacto y comunicación con sus pares (amigos o compañeros), que disponen de tecnologías móviles sofisticadas a través de la cuales pueden crear, compartir y difundir múltiples objetos digitales (fotografías, videos, audios, textos,...). Todo ello está provocando una mutación profunda en las pautas de interacción comunicativa y de consumo de información (Cerezo, 2017) que se evidencia de forma notoria en los estudiantes universitarios.

Mi alumnado actual invierte diariamente muchas horas en actividades realizadas con la tecnología digital: navegar por la web, comunicarse y mantener relaciones sociales en las redes, divertirse y jugar, elaborar contenidos en formato texto, foto o video y compartirlos, intercambiar mensajes, leer noticias, realizar compras online, bajar y subir ficheros de diversa naturaleza, ... En su práctica social con las tecnologías son habilidosos digitalmente, pero, en gran parte de los casos, no disponen de los

De la enseñanza presencial a la docencia digital. Autobiografía de una historia de vida docente. Manuel Area Moreira

Página 16 de 21 
conocimientos, saberes y competencias para hacer un uso culto, crítico y pleno (al menos desde una perspectiva educativa) de toda la potencialidad de conocimiento e información disponible en la Red. Los retos formativos que implica todo ello son abrumadores y requieren el cambio de paradigma educativo al que aludí anteriormente.

Todo ello, nos obliga a los docentes universitarios a tener en cuenta estos profundos cambios culturales de nuestros estudiantes y adecuar las metodologías de enseñanza, los materiales didácticos y los entornos formativos digitales a sus intereses, necesidades y pautas de interacción con la cultura y el conocimiento. De este modo, resultará ineludible a corto y medio plazo que debemos superar modelos de aulas virtuales o entornos formativo concebidas como entornos online cerrados y basados en una concepción expositiva del conocimiento que proporcionan materiales estructurados con muchos textos narrativos similares a los producidos en papel y/o con videolecciones excesivamente largas y sin la suficiente calidad técnica de audio e imagen son materiales didácticos obsoletos y que poco atraerá la atención e interés de nuestros estudiantes.

En este sentido, el modo o formato expresivo más generalizado en el ciberespacio es de microcontenido entendido como "pequeños fragmentos de información digital en un estado permanente de flujo y circulación. Suele tratarse de un tema concreto, limitado en sus dimensiones, que es consumido rápidamente $\mathrm{y}$, a menudo, también limitado por el software o dispositivo para su visualización (tamaño de la pantalla, ancho de banda, navegación, capacidad de atención de los usuarios,...). Por tanto, se basa en la utilización de unidades pequeñas de contenido de aprendizaje y de tecnologías flexibles que permiten a las personas acceder a los mismos más fácilmente en momentos y condiciones específicas cotidianas (p.e., durante el tiempo de descanso o mientras viaja)" (Salinas y Marín, 2014, 47).

Esto significará que los docentes para comunicar adecuadamente, atraer la atención y motivar a nuestros actuales y futuros estudiantes seamos también creadores de microobjetos digitales de aprendizaje en distintos formatos o lenguajes multimedia como son las infografías, los podcast, los mapas conceptuales, los murales o posters digitales, los multimedia interactivos, las líneas de tiempo o videoclips de corta duración, por citar algunos de los recursos más al uso. Todo ello bajo una metodología de aprendizaje activo donde planteemos retos intelectuales y profesionales para que los estudiantes tengan experiencias de aprendizaje valiosas de búsqueda y localización de información, de análisis de la misma así como de construcción y comunicación del conocimiento a través del ciberespacio.

A todo ello hemos de incorporar el conocimiento profesional de docencia online con nuevas metodologías didácticas como son el flipped classroom (o clase invertida), la gamificación del aprendizaje, el aprendizaje ubicuo y móvil, los PLE o entornos personales de aprendizaje (Castañeda y Adell, 2013), los MOOC (Cursos Masivos Online Abiertos) o el aprendizaje adaptativo y el aumentado.

\section{A modo de conclusión inacabada: El viaje continúa}

Todos estos nuevos enfoques o metodologías didácticas digitales suponen un constante desafío para la innovación de la educación superior y, en consecuencia, una nuevo reto profesional docente para los próximos años. La continuación del viaje es utilizar pedagógicamente una nueva generación de entornos digitales del aprendizaje (NGDLE) caracterizados por ser un ecosistema de aplicaciones interconectadas y flexibles que apoyan el aprendizaje a través de cinco dominios clave: interoperabilidad;

De la enseñanza presencial a la docencia digital. Autobiografía de una historia de vida docente. Manuel Area Moreira

Página 17 de 21 
personalización; análisis, asesoramiento y evaluación del aprendizaje; colaboración; accesibilidad y diseño universal (Brown, Dehoney y Millichap, 2015; Feldstein, 2017).

En definitiva, los nuevos entornos o espacios digitales para el aprendizaje anunciados a corto y medio plazo que se basan en la aplicación de la inteligencia artificial, en los formatos narrativos multimedia, en la comunicación social constante y en las analíticas del aprendizaje prometen muchos retos interesantes y estimulan mi curiosidad, por lo que mi biografía como docente universitario que enseña en el ciberespacio todavía es un viaje inacabado (eso quisiera y espero).

Presentación del artículo: 2 de diciembre de 2017

Fecha de aprobación: 8 de enero de 2018

Fecha de publicación: 31 de enero de 2018

Area, M. (2018). De la enseñanza presencial a la docencia digital. Autobiografía de una historia de vida docente. RED. Revista de Educación a Distancia, 56. Consultado el (dd/mm/aaaa) en http://www.um.es/ead/red/56/area.pdf

\section{Financiación}

Esta investigación no ha recibido ninguna subvención específica de los organismos de financiación en los sectores públicos, comerciales o sin fines de lucro.

\section{Bibliografía}

Adell, J. (2004): Nuevas tecnologías en la formación presencial: del curso on-line a las comunidades de aprendizaje. Qurriculum: Revista de Teoría, Investigación y Práctica Educativa, 17, págs. 57-92. Consultado 20/10/2017 en http://revistaq.webs.ull.es/ANTERIORES/numero17/adell.pdf

Aguaded, I. y Cabero, J. (Dir) (2002): Educar en red. Internet como recurso para la Educación, Aljibe, Málaga.

Aguaded, J.I. y Cabero, J. (2013). Tecnologías y medios para la educación en la esociedad. Madrid: Alianza Editorial.

Area, M. (2008): Una breve historia de las políticas de incorporación de las tecnologías digitales al sistema escolar en España. Quaderns digitals: Revista de Nuevas Tecnologías y Sociedad, 51. Número especial XIII aniversario. Consultado el 2/7/2017

http://www.quadernsdigitals.net/index.php?accionMenu=hemeroteca.VisualizaA rticuloIU.visualiza\&articulo_id=10454

Area, M. (2010): "Del HTML a la Web 2.0: autobiografía de una década de docencia universitaria con TIC.” En ROIG, R. y FIORUCCI, M. (Eds.): Claves para la investigación en innovación y calidad educativas. La integración de las tecnologías de la información y la comunicación y la interculturalidad en las aulas. Marfil-ROMATRE, Alcoy, pgs. 55-70.

Area, M. y Adell, J. (2009): “eLearning: Enseñar y aprender en espacios virtuales. En J. De Pablos (Coord): Tecnología Educativa. La formación del profesorado en la era de Internet. Aljibe, Málaga, pags. 391-424

De la enseñanza presencial a la docencia digital. Autobiografía de una historia de vida docente. 
Area, M. y Sanabria, A.L. (2014): Changing the rules: from textbooks to PLEs / Cambiando las reglas de juego: de los libros de texto al PLE. Cultura $y$ Educación, 26 (4), https://doi.org/10.1080/11356405.2014.979068

Area, M.; Borrás, J. y Sannicolás, B. (2015): Educar a la generación de los Millennials como ciudadanos cultos del ciberespacio. Apuntes para la alfabetización digital. Revista Estudios de Juventud, septiembre, 109, 13-32 (monográfico sobre "Juventud Global: Identidades y escenarios de actuación en clave cosmopolita"). Consultado el $10 / 10 / 2017$ en http://www.injuve.es/sites/default/files/cap1_109.pdf

Attwell, G. (2007): Personal Learning Environments - the future of eLearning? eLearning Papers, 2(1). Accesible en https://www.researchgate.net/publication/228350341_Personal_Learning_Envir onments-the_future_of_eLearning (consultado 2/11/2017)

Barberá, E. (2008): Aprender e-learning . Paidós, Barcelona.

Barberà, E.; Bautista, G.; Espasa, A. y Guasch, T. (2006): Portfolio electrónico: desarrollo de competencias profesionales en la Red. RUSC Vol. 3, n. ${ }^{\circ} 2$. Disponible en http://rusc.uoc.edu/ojs/index.php/rusc/article/view/287 (consultado 10/3/2010)

Barberá, E.; Mauri, T.; y Onrubia, J. (Coord) (2008): Cómo valorar la calidad de la enseñanza basada en las TIC. Graó, Barcelona.

Barrera, P., Fernández, C. y Jiménez, F.(2009): “Transición de Docencia Presencial a no Presencial o Semipresencial en un Escenario Heterogéneo". RED, Revista de Educación a Distancia. Número monográfico IX Consultado el 1/10/2017 en http://www.um.es/ead/red/M9

Bartolomé, A. (2008): Entornos de aprendizaje mixto en educación superior. Revista Iberoamericana de Educación a Distancia. v. 11: 1, 2008, pp 15-51. Consultado el 5/9/2017 en http://www.utpl.edu.ec/ried/images/pdfs/volumen11/bartolome.pdf

Bates, A. W. (2015). Teaching in a digital age: Guidelines for designing teaching and learning. British Columbia: BC Open Textbooks. Consultado el 23/09/2017 en http://bit.ly/1T53L46

Benítez, S. y Lemus, M. (2016). La utilización de tecnobiografías como método de estudio de la apropiación de las TIC. $V$ Encuentro Latinoamericano de Metodología de las Ciencias Sociales, Mendoza, Argentina. Consultado el 2/11/2017

http://www.memoria.fahce.unlp.edu.ar/trab_eventos/ev.8187/ev.8187.pdf

Bergmann, J. y Sams (2014): Dale la vuelta a tu clase. Madrid: SM

Brown, M. Dehoney, J. y Millichap, N. (2015): The Next Generation Digital Learning Environment: A Report on Research. EDUCAUSE Learning Initiative, ELI Papers and Reports. Consultado 11/11/2017 en https://library.educause.edu/ /media/files/library/2015/4/eli3035-pdf.pdf

Busse, S.; Ehses, C. y Zech, R. (2000): Collective Research in Autobiography as a Method of the Science of the Subject. Forum: Qualitative Social Research Volume 1, (2) Consultado el 21/9/2017 en http://www.qualitativeresearch.net/index.php/fqs/article/view/1073/2333

Cabero, J. (2006) Bases pedagógicas del e-learning. RUSC. Revista de Universidad y Sociedad del Conocimiento, 3(1) consultado el 8/11/2017 en http://www.uoc.edu/rusc/3/1/dt/esp/cabero.pdf

De la enseñanza presencial a la docencia digital. Autobiografía de una historia de vida docente. Manuel Area Moreira 
Cabero, J. y Gisbert, M. (2005). La formación en Internet. Guía para el diseño de materiales didácticos. MAD; Sevilla

Cabero, J. y Román, P. (2006): E-actividades. Eduforma, Sevilla.

Cerezo, P. (2017): La Generación Z y la información. Revista Estudios de Juventud, no 114. Consultado el 10/10/2017 en http://www.injuve.es/sites/default/files/2017/28/publicaciones/documentos_7._la _generacion_z_y_la_informacion.pdf

Coll, C. y Monereo, C. (eds.) (2008): Psicología de la educación virtual. Morata, Madrid.

Coll, C., Engel, A., Saz, A. y Bustos, A. (2014): Personal learning environments: design and use / Los entornos personales de aprendizaje en la educación superior: del diseño al uso. Cultura y Educación, 26 (4). https://doi.org/10.1080/11356405.2014.985935

Duart, J.M. y Sangrá, A. (Comp.) (2000): Aprender en la virtualidad. Gedisa, Barcelona.

Feldstein, M. (2017): What Is the Next Generation?. EDUCAUSE review july/august 2017, 38-44, Consultado 12/11/2017 en https://er.educause.edu/ /media/files/articles/2017/7/erm17414.pdf

Ferrarotti, F. (2007): Las historias de vida como método. Convergencia. Revista de Ciencias Sociales, vol. 14, núm. 44, pgs. 15-40 Consultado el 1/10/2017 en http://www.redalyc.org/articulo.oa?id=10504402

Fraga, F. y Gewerc, A. (2009): E-portafolios.-La búsqueda de un software coherente con la propuesta de enseñanza. Red U - Revista de Docencia Universitaria. Número monográfico III. Portafolios electrónicos y educación superior en España (en coedición con RED) http://www.um.es/ead/Red_U/m3/ (22/3/2010)

García Aretio, L. (Coord) (2007): De la educación a distancia a la educación virtual. Ariel, Barcelona

Gómez Ortega, J. (Dir.) (2016): UNIVERSITIC 2016. Análisis de las TIC en lasUniversidades Españolas. Madrid, Crue Universidades Españolas. Consultado el 29/10/2017 en http://www.crue.org/Documentos\%20compartidos/Publicaciones/Universitic/U NIVERSITIC\%202016_versi\%C3\%B3n\%20digital.pdf

Goodson, I. (coord.) (2004). Historias de vida del profesorado. Barcelona: Octaedro.

Gros, B. (2002). Constructivismo y diseños de entornos virtuales de aprendizaje. Revista de Educación, 328, pgs. 225-247

Hernández, F. y Rifà, M. (Coords.). (2011). Investigación autobiográfica y cambio social. Barcelona: Octaedro

Hernández, F.; Sancho, J.M ${ }^{\mathrm{a}}$ y Rivas, I. (2011) Historias de Vida en Educación. Biografías en contexto. Esbrina-Cuadernos de Recerca 4, Universitat de Barcelona, Consultado el 1/11/2017 en http://hdl.handle.net/2445/15323

Johnson, L., Adams, S., Cummins, M., Estrada, V., Freeman, A., y Hall, C. (2016). NMC Horizon Report: 2016. Higher Education Edition. Austin, Texas: The New MediaConsortium. Consultado el 1/10/2017 en http://cdn.nmc.org/media/2016nmc-horizon-report-he-EN.pdf\#page30

Mauri, T. y Onrubia, J. (2008): El profesor en entornos virtuales. Condiciones, perfil y competencias. En C. Coll y C. Monereo (eds.)

Medrano, C. (Coord) (2007): Las historias de vida. Implicaciones educativa. Alfagrama, Buenos Aires.

De la enseñanza presencial a la docencia digital. Autobiografía de una historia de vida docente. Manuel Area Moreira

Página 20 de 21 
Monereo, C.; Monte, M. y Andreucci, P. (2015): La gestión de incidentes críticos en la universidad. Narcea, Madrid.

Osorio, L. A. (2011): Ambientes híbridos de aprendizaje. Actualidades Pedagógicas, 58 julio-diciembre del 2011, pgs. 29-44. Consultado el 21/10/2017 en http://revistas.lasalle.edu.co/index.php/ap/article/viewFile/529/449

Revuelta, F.I. y Pérez, L. (2009): Interactividad en los entornos de formación on-line. Editorial UOC, Barcelona.

Rivas, I. y Herrera, D. (Coord) (2009): Voz y educación. La narrativa como enfoque de interpretación de la realidad. Barcelona, Octaedro.

Rivas, I.; Leite, A. y Prados, M. E. (Coord) (2014): Profesorado, escuela y diversidad. La realidad educativa desde una mirada narrativa. Málaga, Ediciones Aljibe.

Rivera-Vargas; Alonso-Cano y Sancho-Gil (2017): Desde la educación a distancia al eLearning: emergencia, evolución y consolidación. Revista Educación y Tecnología, $6 \mathrm{n}^{\circ} 10$. Vol 1, pp 1-13.

Salinas, J (2004): Cambios metodológicos con las TIC. Estrategias didácticas y entornos virtuales de enseñanza-aprendizaje. Bordón, 56 (3-4), pgs. 469-481

Sancho, J.M ${ }^{a}$ (2013): Una propuesta para valorar el trabajo de los estudiantes más allá de los formatos técnicos. En Paredes, J.; Hernández, F., y Correa, J. M. (eds.) (2013). La relación pedagógica en la universidad, lo transdisciplinar y los estudiantes. Desdibujando fronteras, buscando puntos de encuentro. Madrid: Depósito digital UAM. Consultado el 13/10/2017 en http://hdl.handle.net/10486/13152

Siemens, G, Gasevic, D \& Dawson, S (eds) 2015, Preparing for the digital university: a review of the history and current state of distance, blended, and online learning. University of Edinburgh. Consultado el 1/10/2017 en http://www.research.ed.ac.uk/portal/files/21130003/PreparingDigitalUniversity. pdf

Sonlleva. M., Torrego, A. y Martínez, S. (2017): Es una locura vivir sin Facebook ni WhatsApp": la huella tecnológica en el docente en formación. edmetic, Revista de Educación Mediática y TIC. 6(2), pgs. 255-276. Cosultado el 7/11/2017 en doi: https://doi.org/10.21071/edmetic.v6i2.6935

Suárez, C. y Gros, B. (2013): Aprender en red. De la interacción a la colaboración. Editorial UOC, Barcelona

Varios (2014): Blended and Flipped: Exploring New Models for Effective Teaching \& Learning. Faculty Focus, Special Report, Magna Publication, Consultado el $1 / 10 / 2017$

http://www.brandeis.edu/teaching/BlendedandFlipped.pdf

Zapata, M. (2008). "Un cuarto de siglo de ayuda pedagógica en ordenadores y en redes. De la EAO-CAI a los objetos de aprendizaje, al diseño instruccional y a los patrones de e-learning". Quaderns Digitals, $\mathrm{n}^{\circ}$ 51. Número especial XIII aniversario. Consultado el 9/9/2017 http://dialnet.unirioja.es/servlet/articulo?codigo $=2565991$.

Zawacki-Richter, O. y Anderson, T. (2014): Online Distance Education. Towards a Research Agenda. AU Press, Athabasca University. Consultado el 1/10/2017 en http://www.aupress.ca/books/120233/ebook/99Z_Zawacki-

Richter_Anderson_2014-Online_Distance_Education.pdf

De la enseñanza presencial a la docencia digital. Autobiografía de una historia de vida docente. 